\title{
La famille Amrouche, dossier coordonné par Hervé Sanson
}

\section{Claudia Mansueto}

\section{(2) OpenEdition}

10 Journals

\section{Édition électronique}

URL : http://journals.openedition.org/studifrancesi/5798

DOI : 10.4000/studifrancesi.5798

ISSN : 2421-5856

Éditeur

Rosenberg \& Sellier

\section{Édition imprimée}

Date de publication : 1 septembre 2011

Pagination : 470-471

ISSN : 0039-2944

\section{Référence électronique}

Claudia Mansueto, « La famille Amrouche, dossier coordonné par Hervé Sanson », Studi Francesi [En ligne], 164 (LV | II) | 2011, mis en ligne le 30 novembre 2015, consulté le 09 janvier 2021. URL : http:// journals.openedition.org/studifrancesi/5798; DOI : https://doi.org/10.4000/studifrancesi.5798

Ce document a été généré automatiquement le 9 janvier 2021.

\section{(c) (i) (2)}

Studi Francesi è distribuita con Licenza Creative Commons Attribuzione - Non commerciale - Non opere derivate 4.0 Internazionale. 


\title{
La famille Amrouche, dossier coordonné par Hervé Sanson
}

\author{
Claudia Mansueto
}

\section{RÉFÉRENCE}

La famille Amrouche, dossier coordonné par Hervé SANSON, «Expressions maghrébines» vol. 9, n 1, été 2010, pp. 1-219.

1 Ce numéro d'«Expressions maghrébines» est entièrement dédié à la famille Amrouche. Le dossier comprend cinq sections: une brève introduction d'Hervé Sanson qui soumet à l'attention du grand public l'extraordinaire aventure littéraire de Taos et Jean Amrouche; «Articles» (pp. 9-100) qui présente les caractéristiques idéologiques et culturelles des deux célèbres intellectuels maghrébins; «Entretiens et hommages» (pp. 103-145) qui explore l'intimité des frères Amrouche à l'aide des souvenirs des amis qui ont vécu avec eux; «Documents et textes inédits» (pp. 149-175) et «Varia» (pp. 179-219) qui proposent des contributions intéressantes sur le panorama littéraire maghrébin.

2 La section «Articles» comprend six contributions: «Si Amrouche existe, c'est à lui que nous le devons...... Jean Amrouche-Armand Guibert, une amitié créatrice de Guy DUGAS (pp. 9-25); Du poétique au politique. Jean Amrouche compagnon de route d'André Gide de Pierre MASSON (pp. 27-39); Jean Amrouche and the Translation of Chants berbères de Kabylie: the Retrieval and Re-Creation of Collective Memory d'Alexandra ORMEROD (pp. 41-56); Jean Amrouche: Jugurtha ou le témoin premier d'Hervé SANSON (pp. 57-70); Jacinthe noire de Taos Amrouche: un jeu avec l'horizon d'attente d'Ada RIBSTEIN (pp. 71-79); L'Amant imaginaire de Taos Amrouche: roman ou journal intime simulé? de Mokhtar EL MAOUHAL (pp. 81-92).

3 Le premier article présente l'intense et orageuse amitié qui liait Jean Amrouche à Armand Guibert: unis par les mêmes aspirations et encombrés par les mêmes «frustrations éducatives et religieuses» (p.10), Amrouche et Guibert seront deux interprètes privilégiés des difficiles années quarante; ces dernières constituant une période de choix irréversibles et de changements radicaux. À travers l'analyse d'une 
amitié tourmentée et complexe, Guy DUGAS présente les traits les plus significatifs du caractère du jeune Jean Amrouche: ambitieux, inquiet, juge inflexible et critique, l'écrivain kabyle était obsédé par l'idée de rassembler définitivement les lambeaux de son identité déchirée. Dugas souligne en particulier l'enthousiasme de l'auteur maghrébin au moment où Amrouche décide de s'occuper de la réédition des Chants Berbères de Kabylie (1947): recréer une «beauté éparse ou mal comprise» (p. 20) aidait Amrouche à sortir définitivement de la «figure du bâtard culturel» (p. 20), kaléidoscope désordonné de cultures et d'identités contradictoires. Le texte de Dugas insiste sur la modernité de Jean Amrouche; un artiste qui essayait de défendre, à travers la conservation du patrimoine poétique kabyle, l'endroit le plus pur et le plus sensible de son âme, le coin le moins menacé par la vague incontrôlable de son malaise existentiel. L'article qui suit, signé Pierre MASSON, permet d'approcher les raisons qui ont poussé Amrouche à sortir définitivement de la tour d'ivoire de la contemplation poétique pour entrer dans la difficile arène politique des années quarante. Masson souligne comment Amrouche, fervent admirateur de Gide et spectateur ému des événements algériens contemporains, comprendra progressivement que seuls l'engagement et l'intervention active dans le quotidien contribuent à résoudre les drames humains. Le critique met en évidence la crise intérieure qui tourmentait Jean Amrouche: exemple d'une humanité incertaine et sans valeurs, le poète maghrébin précipite dans le désespoir le plus absolu quand il comprend que l'isolement poétique et spirituel de Claudel ne donne que l'illusion de la satisfaction; c'est la participation à la vie réelle qui donne un sens à l'existence humaine. Masson, en citant Amrouche, souligne que «le temps du pur poète est passé. Celui des lois, d'un pays à reconstruire dans sa complexité, est venu» (p. 38). Le troisième article suit le parcours inauguré par les textes précédents. Ormerod, en effet, souligne comment, à travers la traduction des chants kabyles, Amrouche, désormais convaincu de devoir défendre l'identité de son peuple avec l'arme de l'écriture, contribue à sauver un patrimoine culturel que la modernité ignorante et incontrôlable aurait sans aucun doute écrasé. Masson met en évidence l'enthousiasme de Jean Amrouche, auteur d'une extraordinaire aventure culturelle finalisée à défendre la poésie des ancêtres pour assurer «its continuation into the future» (p. 43). Traducteur des poèmes kabyles oraux en français, Amrouche est, comme le souligne ORMEROD, "participator in the promotion and circulation of these narratives and as such, custodian and disseminator of collective memory» (p. 47) parce qu'il protège et réinterprète le patrimoine identitaire d'une culture souterraine, d'une vitalité littéraire qui animait ses souvenirs d'enfance. Le but de cet article est de mettre en évidence «a spiritual force driving the creative impetus for his poetry and his ancestors' songs» (p.45), le sentiment intérieur de Jean Amrouche qui nous explique la raison pour laquelle il avait décidé de poursuivre cet ambitieux projet littéraire et humain: «Je n'ai rien dit qui fût à moi. Je n'ai rien dit qui fût de moi. Ah! Dites-moi l'origine des paroles qui chantent en moi! Est-ce la main d'un Ange présente et absente? Est-ce la main d'un Dieu veillant au-delà de moi-même? De quoi sont-elles messagères? De qui suis-je le messager?» (pp. 45-46). Après avoir compris la valeur de ses origines kabyles, Amrouche décidera d'utiliser son génie artistique pour contribuer à la lutte contre toute forme de colonisation occidentale. L'article d'Hervé SANSON rend compte de la mosaïque que l'identité hybride de Jean Amrouche constitue, en soulignant que le but de l'Éternel Jugurtha (1946), l'essai le plus complexe de l'écrivain, est de montrer, sans masque ni mensonge, la véritable identité kabyle de l'intellectuel, une voix berbère spectrale qui parle à travers l'utilisation de la langue française. Il souligne également 
que la découverte des «identités multiples qui composent la complexité-Amrouche» (p. 61) a aidé le poète maghrébin à comprendre l'importance de la liberté de son peuple: l'autonomie de la terre algérienne était la conditio sine qua non pour dissiper définitivement le brouillard qui couvrait son identité. Si l'article de Sanson explique le difficile parcours de Jean Amrouche à «la reconquête d'anima» (p. 66), la contribution d'Ada RIBSTEIN est finalisée à présenter un autre membre de la famille: Taos. Personnalité hybride, «mélange de force et fragilité» (p. 123), comme le soulignera sa fille Laurence interviewée par Denise Brahimi, Taos Amrouche est un des intellectuels les plus subversifs du panorama littéraire francophone des années quarante. L'auteur met en évidence la modernité de l'œuvre littéraire de Taos Amrouche en analysant un de ses chef-d'œuvres: Jacinthe noire (1947). Ribstein explique que la structure narrative de Jacinthe noire est révélatrice de la personnalité de l'écrivain maghrébin: la totale subversion des codes narratifs, le récit nébuleux, le choix de privilégier le subjectif à la vérité objective, sont des miroirs qui renvoient au lecteur l'image d'une femme ambiguë et hybride. En citant Afifa Bererhi, Ada Ribstein souligne que la «démultiplication/diffraction du Je, le récit autobiographique» (p. 74) ne sont que les symboles extérieurs d'une littérature orientée à privilégier la vérité subjective de l'instant plutôt que l'universalité de canons atemporels. Taos Amrouche est le sujet principal du dernier article de Mokhtar el MAOUHAL. À travers l'analyse de L'Amant imaginaire (1975), il met surtout en évidence l'ambiguïté du récit: «S'agit-il d'un journal authentique ou d'un journal simulé? Est-il question d'un journal ou d'un roman?» (p. 82). Le halo de mystère qui couvre toute la production littéraire de Taos Amrouche est symptomatique de sa personnalité mimétique; un mimétisme intérieur qui parle à travers les voix de ses personnages littéraires. Pour soutenir cette interprétation, le critique souligne la valeur presque prophétique des paroles d'Aména, protagoniste de L'Amant imaginaire et double de Taos Amrouche.

En conclusion, cette section de la revue «Expressions maghrébines» a le mérite d'avoir contribué à souligner la modernité intellectuelle et idéologique des frères Amrouche. Précurseurs de la poétique de l'hybride et du pluriel identitaire postcolonial, Taos et Jean Amrouche, déchirés entre l'être et l'avoir, l'Un et le multiple, le masque et la vérité, représentent les incertitudes de l'homme contemporain, un homme obsédé par ses doutes et tourmenté par ses aspirations, ou mieux par cette «tension vers...» (p. 119) qui résume toute la grandeur de la vie. 